\title{
Motion Analysis and Power Responses of a Single-buoy Wave Energy Converter Consisting of Double Hydraulic Cylinders
}

\author{
Biao Li, Hongtao Gao* \\ Dalian Maritime University, Dalian, Liaoning, China \\ gaohongtao@dlmu.edu.cn.
}

Keywords: Wave energy; Buoy; Response; Conversion power; Heaving-pitching.

\begin{abstract}
A single-buoy wave energy converter (WEC) consisting of double hydraulic cylinders is designed to promote the conversion power of traditional oscillating buoy WEC by absorbing the energy of pitching motion. On the condition of linear, regular waves, the nonlinear coupled equations of the pitching-heaving motions of buoy and energy conversion expressions are deduced with a wave incident angle of zero. Then the motion characteristics and power responses are analyzed. Calculations indicate that the heaving and pitching motions can be expressed as approximate simple harmonic motions with wave period. The wave circular frequency and draft of buoy have obvious influences on the amplitude-frequency responses. When the draft is larger than $1.0 \mathrm{~m}$, the curves of heaving motion exhibit single-peak patterns and the peak values are discovered at the circular frequency of about $2.5 \mathrm{rad} / \mathrm{s}$. The amplitude-frequency responses of pitching motion share the similar trends, and the peak amplitudes change regularly with draft and circular frequency. The heaving motion and pitching motion together influence the conversion power. For the cases in this paper, larger draft can increase the conversion power on the whole. According to the power contribution of pitching motion, the rates of conversion power of pitching motion usually exceed 20\%. Therefore, the conversion power of traditional oscillating buoy WEC is expected to be improved effectively by absorbing the energies of multiple freedoms.
\end{abstract}

\section{INTRODUCTION}

The single-buoy devices are the simplest point-absorber wave energy converters (WECs) for deployment offshore to harvest boundless and high-density ocean wave energy [Falcão A.F., 2010] [Behrens S., 2012] [Stansell P., 2013]. In traditional design of single-buoy WECs, the oscillating buoy is one of the most common and studied types, which performs heaving oscillations relative to a strut connected to an anchor on the sea bed [Budal K., 1982]. In fact, according to the theory of multi-rigid-body system dynamics [Wittenburg J., 2007] [Sun Z.C., 2009], the motion of buoy on wave are carried out in a three dimensional system with six-degree-freedom (rolling, pitching, yawing, surging, swaying and heaving). It is inferred that more motion energy of freedoms might be absorbed by optimum design of device.

This paper introduces a new type of single-buoy WEC consisting of double hydraulic cylinders (Figure 1) to capture wave energy as efficient as possible. The buoy partially submerged in the seawater to gather wave energy. The hydraulic cylinders are served as power take-off (PTO) system to extract and convert energy. The damping plate with very large weight and inertia is suspended in seawater, so its motion can be ignored during normal operation. The spring attached to buoy and damping plate, store energy during half a wave cycle and simultaneously provides the buoy with part of restoring force. 


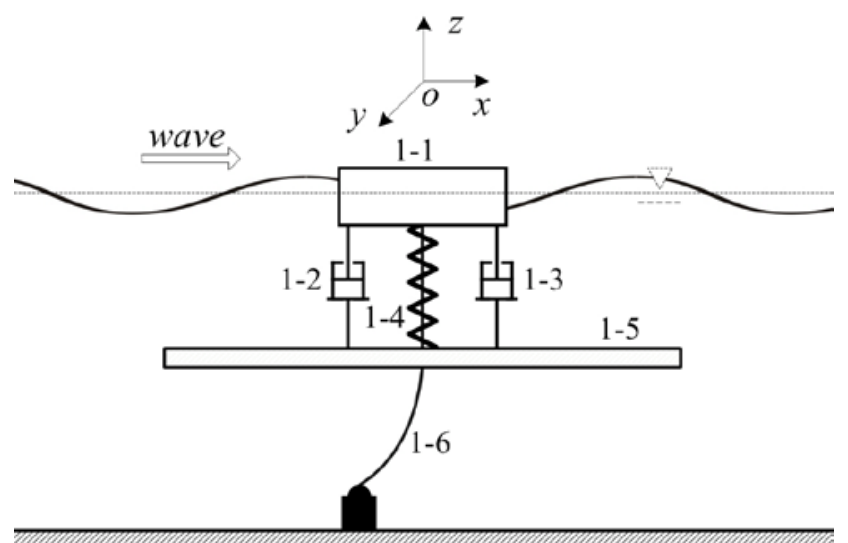

Figure 1. Configuration diagram of the wave energy converter

(1-1: buoy; 1-2, 1-3: hydraulic cylinders; 1-4: spring; 1-5: damping plate; 1-6: anchor chains)

The incidence angle of wave usually approaches zero under the action of waves, wind and anchor chains. Therefore, the motion of the buoy can be simplified into the coupled pitching-heaving motion. The work here present focuses on the establishment of buoy motion model by the derivation of the coupled heaving-pitching equations. Based on the model, the motion and power responses are analyzed.

\section{MATHEMATICAL MODEL}

\subsection{Coupled equations of pitching-heaving motion}

To simplify the calculation process, it is assumed that the weight of buoy is uniformly distributed throughout and the WEC is operated in an open sea environment. The motion of the buoy is resolved into the translation of the center of mass and the rotation around the hinge point of buoy and spring [Wang G.B., 2014]. Based on simplified cases where water waves are treated as linear, regular waves, the two motions are represented in a set of equations by the Newton-Euler method.

The equations (1) of heaving motion are derived according to Newton's second law.

$\left(m+m_{A Z}\right) \cdot \ddot{z}=-m g+F_{F}+F_{H Z}+F_{S}+\left(F_{C 1}+F_{C 2}\right)+F_{K}$

The equations (2) of pitching motion are derived according to moment of momentum theorem.

$$
\begin{aligned}
\left(I_{Y Y}+I_{A Y Y}\right) \cdot \ddot{\theta}= & {\left[\left(F_{H Z}{ }^{(L)}+F_{S}^{(L)}\right)-\left(F_{H Z}{ }^{(R)}+F_{S}{ }^{(R)}\right)\right] \cdot(L / 4) } \\
& +\left(F_{C 1}-F_{C 2}\right) \cdot(L / 2)
\end{aligned}
$$

Where, $m$ is the mass of buoy, $m_{A Z}$ is the added mass components. $F_{H Z}$ is the hydrodynamic force component, $F_{F}$ is buoyancy of buoys, $F_{S}$ is hydrostatic restoring force. $F_{H Z}{ }^{(L)}$ and $F_{H Z}{ }^{(R)}$ are the hydrodynamic force components in the left and right half of buoy. $F_{S}{ }^{(L)}$ and $F_{S}{ }^{(R)}$ are the hydrostatic restoring forces in the left and right half of buoy. $I_{Y Y}$ and $I_{A Y Y}$ are the moment of inertia and added moment of inertia to Y-axis. $\theta$ and $\ddot{\theta}$ are the pitching angle and angular acceleration. $F_{C 1}$ and $F_{C 2}$ are the forces of the hydraulic cylinder piston rods (hydraulic cylinder 1-2 and 1-3). $F_{K}$ is spring force. $L$ is length of buoy.

The related forces are expressed as follow.

$$
\begin{aligned}
& F_{F}=m g \\
& F_{S}=-\rho g S_{W} \cdot z[\text { Mc Cormick M.E., 1976] } \\
& F_{C 1}+F_{C 2}=2 C \dot{z}, \quad F_{C 1}-F_{C 2} \approx L \dot{\theta} \\
& F_{H}=\operatorname{Re}\left[F_{0} \exp (-i \omega t)\right]=F_{0} \cos \omega t \text { [Wu B.J., 2013] (6) } \\
& F_{K}=-K z
\end{aligned}
$$


Where $S_{W}$ is the mean wetted surface area of buoys in still water. $C$ is linear damping coefficient of hydraulic cylinders. $K$ is spring constant.

Then the second order differential equations of heaving-pitching motion are derived as follow.

$\left(m+m_{A Z}\right) \cdot \ddot{z}-2 C \cdot \dot{z}-\left(\rho g S_{W}+K\right) \cdot z=F_{Z 0} \cos \omega t$

$\left(I_{Y Y}+I_{A Y Y}\right) \cdot \ddot{\theta}-\frac{1}{2} C L^{2} \cdot \dot{\theta}+\frac{1}{8} \rho g S_{W}{ }^{(L)} L^{2} \cdot \theta$

$=\frac{1}{4} F_{H 0}{ }^{(L)}[\cos \omega t-\cos (\omega t-\omega \cdot \Delta t)]$

Here $\Delta t$ represents the travel time of wave passing the half length of buoy.

\subsection{Wave energy conversion power}

The wave energy conversion power of PTO system is expressed by the formula (10).

$P_{C 1}=C \cdot\left(\dot{z}+\frac{L}{2} \dot{\theta}\right)^{2}, \quad P_{C 2}=C \cdot\left(\dot{z}-\frac{L}{2} \dot{\theta}\right)^{2}$

Here $P_{C 1}$ is the power of hydraulic cylinder 1-2, $P_{C 2}$ is the power of hydraulic cylinder 1-3.

The time-averaged power $\bar{P}_{C}$ converted by PTO system is:

$$
\begin{aligned}
\bar{P}_{C} & =\bar{P}_{C 1}+\bar{P}_{C 2}=\frac{1}{T} \int_{0}^{T} C \cdot\left[\left(\dot{z}+\frac{L}{2} \dot{\theta}\right)^{2}+\left(\dot{z}-\frac{L}{2} \dot{\theta}\right)^{2}\right] \cdot d t \\
& =\frac{2 C}{T} \int_{0}^{T}\left(\dot{z}^{2}+\frac{L^{2}}{4} \dot{\theta}^{2}\right) d t
\end{aligned}
$$

Where $T$ is wave period.

\section{CALCULATION RESULTS AND ANALYSIS}

The amplitude-frequency response and wave energy conversion power are analyzed using above differential equations of heaving-pitching motion. The main parameters of WEC and sea condition are shown in Table 1 and Table 2.

Table 1. Main parameters of WEC

\begin{tabular}{ll}
\hline Structure Parameter & Value \\
\hline Shape of buoy & Cuboid \\
Length of buoy/L & $6.0 \mathrm{~m}$ \\
Width of buoy/ B & $2.0 \mathrm{~m}$ \\
Height of buoy $/ Z$ & $2.0 \mathrm{~m}$ \\
Damping coefficient $/ C$ & $3500 \mathrm{Ns} / \mathrm{m}$ \\
Spring constant $/ K$ & $120 \mathrm{kN} / \mathrm{m}$ \\
\hline
\end{tabular}

Table 2. Conditions of China Yellow Sea and Bohai Sea [Yin W.Y., 2006] [Sun L.J., 2004]

\begin{tabular}{ll}
\hline Parameter & Value \\
\hline Wave direction & $0^{\circ}$ \\
Wave height $/ H$ & $1.2 \mathrm{~m}$ \\
Wave period/T & $2.5 \mathrm{~s}-5.0 \mathrm{~s}$ \\
Wave circular frequency $/ \omega$ & $1.26 \mathrm{rad} / \mathrm{s}-2.51 \mathrm{rad} / \mathrm{s}$ \\
Depth $/ d$ & $40 \mathrm{~m}$ \\
Sea-water density $/ \rho$ & $1025 \mathrm{~kg} / \mathrm{m}^{3}$ \\
\hline
\end{tabular}

Figure 2 and Figure 3 show the displacement of heaving motion $z(t)$ and angle of pitching motion $\theta(t)$ of buoy according to our calculative model. The results show that both the motions of heaving and pitching can be easily expressed as approximate simple harmonic motions with wave period. Therefore, the analysis of buoy motions can be achieved through the discussion of amplitude-frequency response characteristic. 


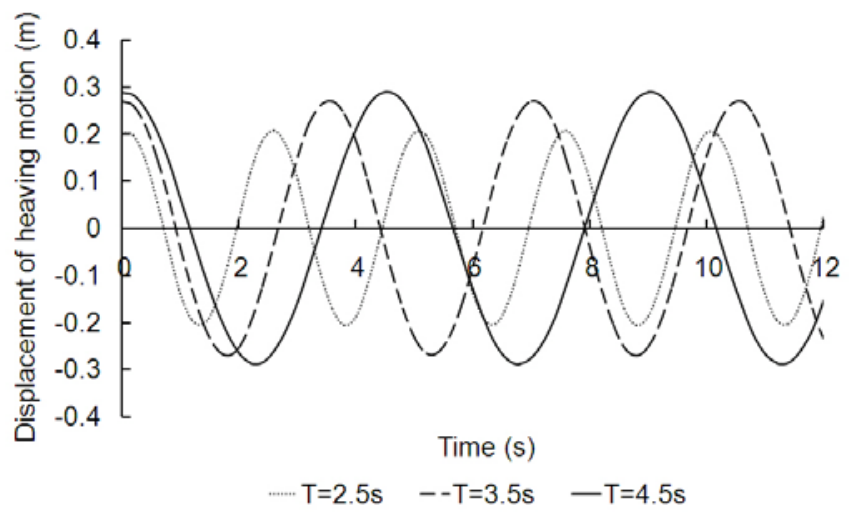

Figure 2. Fitted curves of displacement of heaving motion of buoy at the draft of $1.0 \mathrm{~m}$

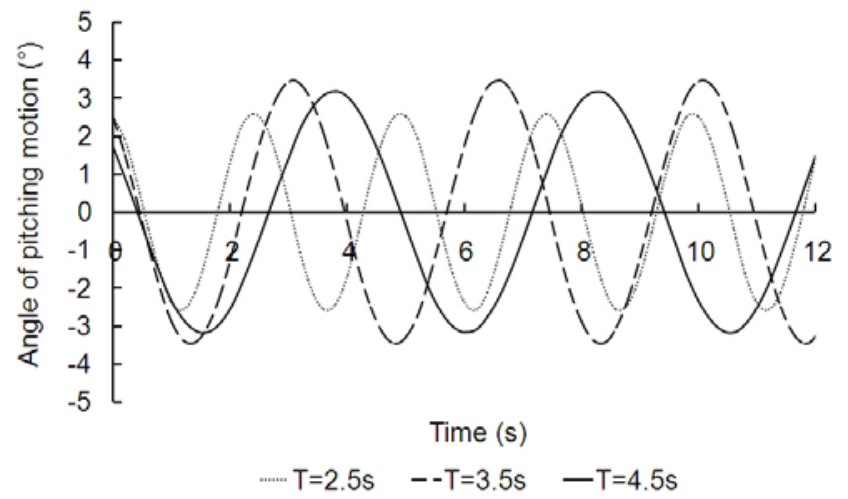

Figure 3. Fitted curves of angle of pitching motion of buoy at the draft of $1.0 \mathrm{~m}$

\subsection{Amplitude-frequency response characteristics with draft of buoy}

Figure 4 shows the amplitude-frequency response of heaving motion with draft of buoy. In the low circular frequency band ( $\omega \leq 2.0 \mathrm{rad} / \mathrm{s}$ ), the amplitudes of heaving motion are generally remained at about $0.28 \mathrm{~m}$ no matter how draft changes. In the intermediate circular frequency band (2.0rad / $\leq \omega \leq 3.2 \mathrm{rad} / \mathrm{s}$ ), the draft have obvious impacts on amplitude-frequency response. When the draft is less than $1.0 \mathrm{~m}$, the amplitudes declined rapidly with circular frequency. When the draft is larger than $1.0 \mathrm{~m}$, the amplitude-frequency responses are changed as single peak curves, and the peak values are found at the circular frequency of about $2.5 \mathrm{rad} / \mathrm{s}$. In the high circular frequency band ( $\omega \geq 3.2 \mathrm{rad} / \mathrm{s}$ ), the amplitudes gradually tend to 0 .

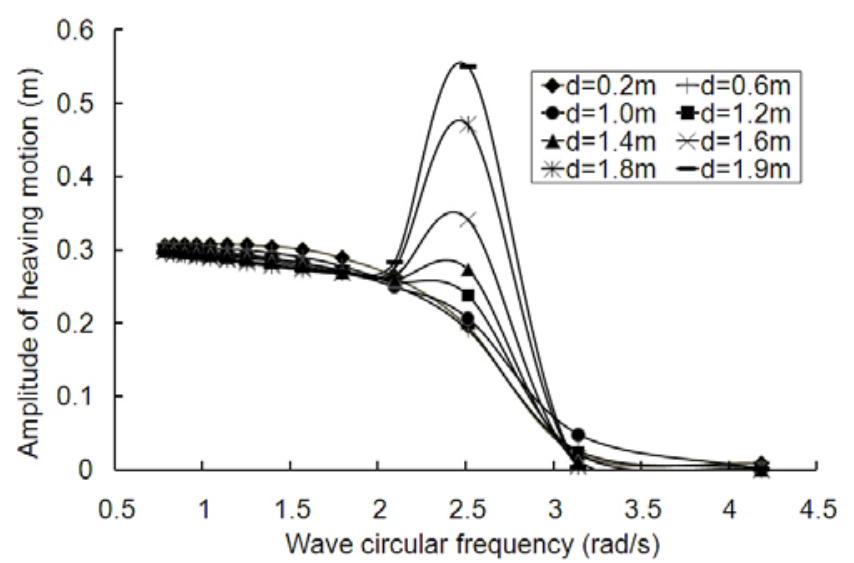

Figure 4. Fitted curves of amplitude-frequency response of heaving motion with draft of buoy

The reason is that the main factor which affects the heaving motion responses is the proximity of the natural frequency of buoys to the wave period [Budal K., 1975].

Figure 5 shows the amplitude-frequency response of pitching angle with draft of buoy. The peak amplitudes of the different cases are discovered within the investigated circular frequency range. The curve changes share the similar trends and patterns. For smaller drafts, the amplitudes are 
always relatively big at a given circular frequency, and the wave circular frequencies corresponding to the peak amplitudes are also big.

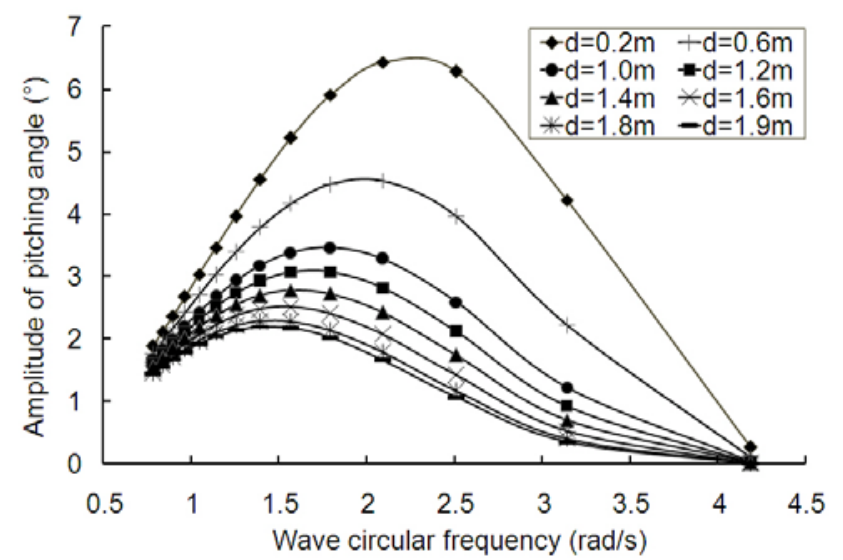

Figure 5. Fitted curves of amplitude-circular frequency response of pitching angle with draft of buoy

\subsection{Conversion power response characteristic with draft of buoy}

Figure 6 shows the conversion power response with draft of buoy. Under the combined action of heaving and pitching motion, the conversion power attain their maximum values in the circular frequency range of $2.2 \mathrm{rad} / \mathrm{s}$ to $2.6 \mathrm{rad} / \mathrm{s}$. When the draft is $1.9 \mathrm{~m}$, the maximum conversion power can reach $6760 \mathrm{~W}$ discovered at the circular frequency of about $2.5 \mathrm{rad} / \mathrm{s}$. In addition, when the draft is $0.2 \mathrm{~m}$, the device also has higher conversion power because of the bigger pitching amplitude. Therefore, the effects of draft on conversion power are significant and complex. For the cases in this paper, larger draft can increase the conversion power on the whole.

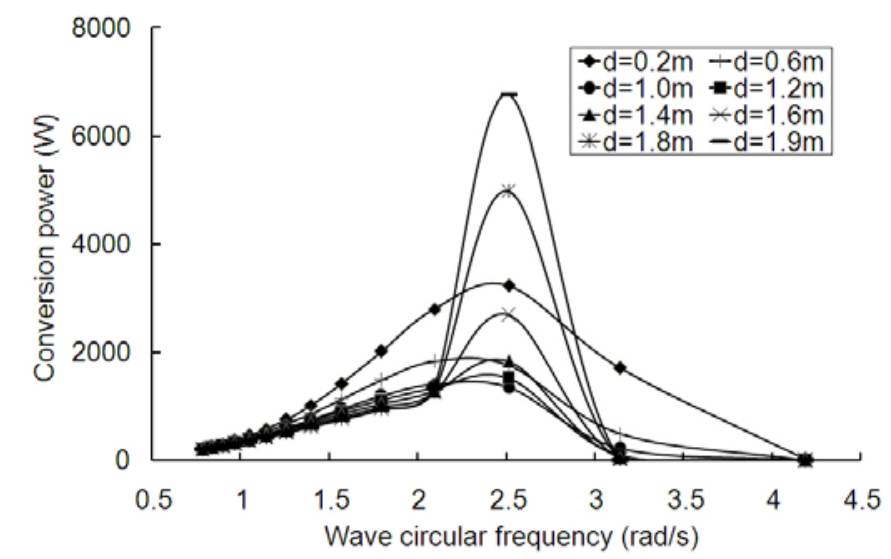

Figure 6. Fitted curves of conversion power response with draft of buoy

\subsection{Promote effect of conversion power}

Figure 7 shows the rate of power of pitching motion with draft of buoy at several common wave periods ( $2.5 s \leq T \leq 5.0 s$ ) which are from China relevant waters [Yin W.Y., 2006] [Sun L.J., 2004]. It is indicated that the rates of conversion power of pitching motion usually exceed $20 \%$. Therefore, the conversion power of traditional oscillating buoy WEC is expected to be improved effectively by absorbing the energies of multiple freedoms. 


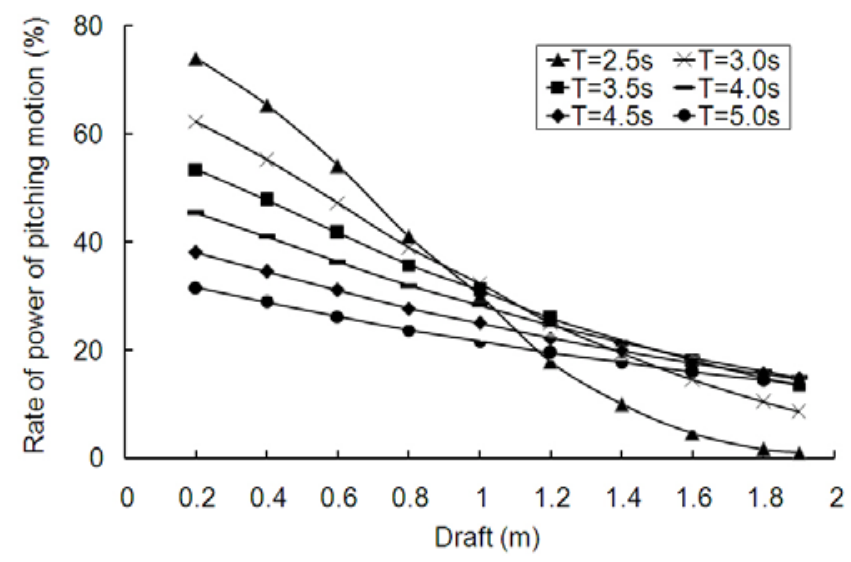

Figure 7. Fitted curves of rate of power of pitching motion with draft at several common wave periods

\section{CONCLUSION}

This paper introduces a single-buoy WEC consisting of double hydraulic cylinders to improve the wave energy conversion performance of the traditional oscillating buoy WEC. On the condition of linear, regular waves, the motion characteristics and power responses are analyzed. The main conclusions are as follows.

(1) The heaving and pitching motions can be easily expressed as approximate simple harmonic motions with wave period.

(2) The amplitude-circular frequency responses of heaving motion are changed as single peak curves when the draft of buoy is larger than $1.0 \mathrm{~m}$ and their peak values are found at the circular frequency of about $2.5 \mathrm{rad} / \mathrm{s}$. The amplitude-frequency responses of pitching motion share the similar trends and patterns that the single peak amplitudes always exist.

(3) The conversion power depends on the coupled characteristic of heaving-pitching motion. Larger draft can increase the conversion power on the whole.

(4) The rates of conversion power of pitching motion usually exceed $20 \%$. The efficient use of energy of pitching motion can promote the conversion power of traditional oscillating buoy WEC effectively.

\section{ACKNOWLEDGEMENTS}

This work was financially supported by the Maritime Safety Administration of the People's Republic of China (2012_26) and the Fundamental Research Funds for the Central Universities (3132014338).

\section{REFERENCES}

[1] Behrens S., Hayward J., Hemer M. 2012. Assessing the wave energy converter potential for Australian coastal regions. RENEWABLE ENERGY. 43: 210-217.

[2] Budal K., Falnes J. 1975. A resonant point absorber of ocean-wave power. Nature. 256: 478479.

[3] Budal K., Falnes J. 1975. Power generation from ocean waves using a resonant oscillating system. Marine Science Communication. 1: 269-288.

[4] Budal K., Falnes J., Iversen L.C., et al. 1982. The Norwegian wave-power buoy project. In: Proceedings of the 2nd International Symposium on Wave Energy Utilization, Trondheim, Norway. 323-344. 
[5] Falcão A.F., de O. 2010. Wave energy utilization: a review of the technologies. Renewable Sustainable Energy Reviews. 14: 899-918.

[6] Mc Cormick M.E. 1976. A modified linear analysis of a wave-energy conversion buoy. Ocean Engineering. 3 (3): 133-144.

[7] Stansell P., Pizer D.J. 2013. Maximum wave-power absorption by attenuating line absorbers under volume constraints. Applied Ocean Research. 40: 83-93

[8] Sun L.J., Gao C. 2004. Statistics and analysis on storm and climate of Yellow Sea and the Bohai Sea. World Shipping. 27 (3): 18-19.

[9] Sun Z.C., Wang H.Y. 2009. Coupling calculation of hinged multi-body floating structure by analytical method. Journal of Ship Mechanics. 13 (1): 34-40.

[10]Wang G.B., Gou Y., Teng B., et al. 2014. Motion responses of hinged multiple floating bodies under regular wave action. Journal of Dalian University of Technology. 54 (6): 618-625.

[11]Wittenburg J. 2007. Dynamics of Multibody Systems. Berlin: Springer International Publishing AG. 223.

[12]Wu B.J., Wang X., Diao X.H., et al. 2013. Response and conversion efficiency of a wave energy device consisting of double cylindrical floats with two degrees of freedom. SCIENTIA SINICA Physica, Mechanica \& Astronomica. 43 (8): 978-986.

[13]Yin W.Y., Zhang Y.N. 2006. Statistical analysis of wind and features at Bohai straits. Journal of Dalian Maritime University. 32 (4): 84-88. 